\title{
Effects of takeaway food consumption on postprandial lipaemia and diet quality: a study on cardiovascular disease risk
}

\author{
H.G. Janssen and I.G. Davies \\ Nutrition and Health Research Group, Liverpool John Moores University, Faculty of Education, Health \& Community, \\ Barkhill Road, Liverpool, L17 6BS
}

Postprandial lipaemia (PPL) is a risk factor for cardiovascular disease (CVD) due to its atherogenic tendencies ${ }^{(1,2)}$. PPL is characterised by an increase in the concentration of blood triglycerides and the metabolic events that occur following the digestion and absorption of a meal containing fat ${ }^{(3)}$. Consumption of takeaway food (TF) has been on the rise over the past few decades ${ }^{(4)}$, often containing a range of ingredients associated with a risk of developing $\mathrm{CVD}^{(5)}$. To date there have been no studies which compare differences in TF consumption and the effects, if any, on postprandial responses to a high calorie test meal (HCTM). The aim of the present study was to investigate the effect of TF consumption on markers of postprandial lipid metabolism and risk of developing CVD later on in life. The objectives were to identify the dietary patterns of two groups, measure their lipid profiles, and assess whether regular TF consumers (RTFC) had a higher intake of unhealthy foods compared to non-TF consumers (NTFC).

Ethical approval and written informed consent were obtained prior to participation. Thirteen healthy volunteers were split into two groups; RTFC and NTFC. The HCTM was administered and bloods were analysed across a two hour period using Cholestech LDX point of care to assess PPL between groups. Usual dietary intake was assessed by using the EPIC-Norfolk food frequency questionnaire and responses were processed using FETA software. Demographical characteristics and anthropometrics were recorded. All data were analysed using descriptive statistics; SQRT transformations were applied to data not normally distributed. Mixed betweenwithin subjects ANOVA, to compare variables, and independent t-tests, to differentiate nutrient intakes, were carried out between RTFC and NTFC. PPL response was measured as area under the curve and incremental area under the curve according to the trapezoid rule.

\begin{tabular}{|c|c|c|c|c|c|}
\hline Nutrient & RTFC $(\mathrm{N}=6)$ & & NTFC ( & & $P$ value \\
\hline & $\mathrm{M}$ & SD & $\mathrm{M}$ & SD & \\
\hline Energy (kcal) & $3025 \cdot \overline{61}$ & $1899 \cdot 57$ & $1413 \cdot \overline{47}$ & $564 \cdot 19$ & $0 \cdot 054$ \\
\hline Total CHO (g) & $361 \cdot 34$ & $175 \cdot 17$ & $152 \cdot 66$ & 81.79 & $0 \cdot 016$ \\
\hline CHO from sugar (g) & 195.58 & 101.01 & 55.79 & $12 \cdot 28$ & $0 \cdot 019$ \\
\hline Total Fat $(\mathrm{g})$ & $126 \cdot 44$ & 83.60 & 61.86 & $27 \cdot 47$ & 0.079 \\
\hline Saturated FA (g) & $50 \cdot 20$ & $36 \cdot 17$ & $20 \cdot 65$ & $8 \cdot 33$ & 0.058 \\
\hline Protein $(\mathrm{g})$ & $113 \cdot 75$ & $90 \cdot 21$ & $66 \cdot 08$ & $16 \cdot 53$ & $0 \cdot 195$ \\
\hline $\operatorname{NSP}(g)$ & $22 \cdot 40$ & $12 \cdot 43$ & $12 \cdot 13$ & 4.06 & $0 \cdot 101$ \\
\hline Salt g* (from sodium) (g) & 8.97 & $3 \cdot 86$ & $4 \cdot 38$ & 1.41 & 0.032 \\
\hline
\end{tabular}

Notes: $n=$ number of participants. P Value $=$ difference between TF groups. Data was represented as mean (M) and standard deviation (SD). *Salt content was calculated from sodium concentration by multiplying by 2.542

PPL was not significantly different between groups. However, regular TF intake was positively associated with higher intakes of total carbohydrate $(p=0.016)$, sugar $(p=0.019)$ and salt $(p=0.032)$. These findings could be attributed to the TF composition reported as high in fats, salt and sugar ${ }^{(4)}$, or they may be a marker for a high risk diet. In conclusion, a diet high in TF has not been shown to effect PPL in a sample of young adults. However, regular TF consumption has been shown to increase intake of some nutrients which have been associated with CVD risk when consumed in large quantities over a long period of time.

1. Adiels M, Matikainen N, Westerbacka J et al. (2012) Atherosclerosis, 222(1), 222-228.

2. Pirillo A, Norata G \& Catapano A (2014) Curr Med Res Opin, 30(8), 1489-1503.

3. Jackson KG, Poppitt SD \& Minihane AM (2012) Atherosclerosis, 220(1), 22-33.

4. Jaworowska A, Blackham T, Davies I et al. (2013) Nutr Rev, 71(5), 310-318.

5. Jaworowska A, Blackham T, Long R et al. (2014) Nutrition \& Food Science, 44(5), 414-430. 